\title{
PROPANE ON TITAN
}

\author{
H. G. Roe, ${ }^{1,2}$ T. K. Greathouse, ${ }^{1,3}$ M. J. Richter, ${ }^{1,4}$ and J. H. LaCY ${ }^{1,3}$ \\ Received 2003 August 27; accepted 2003 September 18; published 2003 October 9
}

\begin{abstract}
We present the first observations of propane $\left(\mathrm{C}_{3} \mathrm{H}_{8}\right)$ on Titan that unambiguously resolve propane features from other numerous stratospheric emissions. This is accomplished using a $R=\lambda / \delta \lambda \approx 10^{5}$ spectrometer (the Texas Echelon Cross Echelle Spectrograph) to observe propane's $\nu_{26}$ rotation-vibration band near $748 \mathrm{~cm}^{-1}$. We find a best-fit fractional abundance of propane in Titan's stratosphere of $(6.2 \pm 1.2) \times 10^{-7}$ in the altitude range to which we are sensitive (90-250 km or 13-0.24 mbar).
\end{abstract}

Subject headings: infrared: solar system — molecular data — planets and satellites: individual (Titan)

\section{INTRODUCTION}

Titan's thick atmosphere is simultaneously analogous to and extraordinarily different from that of Earth. Both atmospheres are composed mostly of nitrogen. Both atmospheres have a major component that can exist as a solid, liquid, or gas (water on Earth, methane on Titan). Both have similar vertical temperature-pressure structures, although Titan's atmosphere is $\sim 200 \mathrm{~K}$ cooler and is greatly extended relative to Earth because of much lower surface gravity. On Titan a complicated network of photochemical reactions leads from methane $\left(\mathrm{CH}_{4}\right)$ to the formation of numerous heavier hydrocarbons, including propane $\left(\mathrm{C}_{3} \mathrm{H}_{8}\right)$. Measuring the abundances of Titan's hydrocarbons provides an important test of models of Titan's atmospheric chemistry and, more generally, of our fundamental understanding of atmospheric chemical and physical processes.

Photochemical models (e.g., Yung, Allen, \& Pinto 1984; Toublanc et al. 1995; Lara et al. 1996; Banaszkiewicz et al. 2000) predict that propane is formed via $\mathrm{C}_{2} \mathrm{H}_{5}+\mathrm{CH}_{3}+\mathrm{M} \rightarrow \mathrm{C}_{3} \mathrm{H}_{8}+$ $\mathrm{M}$, where $\mathrm{CH}_{3}$ is created by methane photolysis and $\mathrm{C}_{2} \mathrm{H}_{5}$ by $\mathrm{C}_{2} \mathrm{H}_{4}+\mathrm{H}+\mathrm{M} \rightarrow \mathrm{C}_{2} \mathrm{H}_{5}+\mathrm{M}$. The primary removal mechanism for propane is condensation at the cold tropopause, although photolytic destruction and reaction with $\mathrm{C}_{2} \mathrm{H}$ consume $\sim 5 \%-15 \%$ of the propane formed. In the model of Lara et al. (1996), propane condensed onto Titan's surface accounts for $\sim 1 \%$ of the carbon removed from Titan's atmosphere.

Detections of propane's $\nu_{26}$ rotation-vibration band near $748 \mathrm{~cm}^{-1}$ were based on Voyager I and Infrared Space Observatory (ISO) spectra. Voyager spectra had $4.3 \mathrm{~cm}^{-1}$ resolution $\left(R=\lambda / \delta \lambda=170\right.$ at $\left.748 \mathrm{~cm}^{-1}\right)$, and initial claims of a propane abundance of $2 \times 10^{-5}$ (Maguire et al. 1981) were later reduced to $(7 \pm 4) \times 10^{-7}$ (Coustenis, Bezard, \& Gautier 1989). The resolution of these spectra left every band of propane blended with other emission features. At $748 \mathrm{~cm}^{-1}$ the $\nu_{26}$ band of propane was blended with features of acetylene $\left(\mathrm{C}_{2} \mathrm{H}_{2}\right)$ and the derived abundance (or even detection) of propane is strongly dependent on the assumed $\mathrm{C}_{2} \mathrm{H}_{2}$ vertical abun-

\footnotetext{
${ }^{1}$ Visiting Astronomer at the Infrared Telescope Facility, which is operated by the University of Hawaii under cooperative agreement NCC 5-538 with the National Aeronautics and Space Administration, Office of Space Science, Planetary Astronomy Program.

${ }^{2}$ O. K. Earl Postdoctoral Scholar in Planetary Science, Division of Geological and Planetary Sciences, California Institute of Technology, MS 17025, Pasadena, CA 91125; hroe@gps.caltech.edu.

${ }^{3}$ Department of Astronomy, University of Texas at Austin, RLM 15.308, C-1400, Austin, TX 78712; tommyg@astro.as.utexas.edu, lacy@astro.as .utexas.edu.

${ }^{4}$ Department of Physics, University of California at Davis, 1 Shields Avenue, Davis, CA 95616; richter@physics.ucdavis.edu.
}

dance profile. Spectra taken by ISO were of higher resolution $\left(R \approx 1850\right.$ at $\left.748 \mathrm{~cm}^{-1}\right)$ but were still unable to resolve propane from acetylene. From these data Coustenis et al. (2003) derived a nominal propane abundance of $(2 \pm 1) \times 10^{-7}$, although they acknowledged the substantial difficulties of disentangling propane emission from that of acetylene.

\section{OBSERVATIONS}

Observations were made at the $3.0 \mathrm{~m}$ NASA Infrared Telescope Facility (IRTF) using the Texas Echelon Cross Echelle Spectrograph (TEXES), a mid-infrared high-resolution grating spectrograph (Lacy et al. 2002) on 2002 December 13, 14, and 20 UT in several overlapping spectral settings around propane's $\nu_{26}$ fundamental vibration at $748 \mathrm{~cm}^{-1}$. At this wavenumber a single spectral setting covers $\sim 5 \mathrm{~cm}^{-1}$ at a resolution of $R \approx$ $10^{5}$ with slight gaps between spectral orders. The strongest $\nu_{26}$ propane lines are blocked by telluric absorption at $748.3 \mathrm{~cm}^{-1}$. The diffraction limit of IRTF at these wavelengths and Titan's diameter are both $\approx 0.9$. With a slit width of 1.5 , our data represent a disk average spectrum of Titan. The reduced spectrum from each night, corrected for the appropriate Doppler shift, is shown in Figure 1 along with the final combined spectrum. All data were reduced using the standard pipeline reduction detailed in Lacy et al. (2002). Telluric transmission was corrected with Callisto on the first two nights and the BecklinNeugebauer object (Becklin \& Neugebauer 1967) on the third night. We flux-calibrated using observations of $\beta$ Gem (Cohen et al. 1995) on the final night and estimate that this calibration is accurate to $10 \%-20 \%$.

\section{RADIATIVE TRANSFER MODELING}

We use a new line-by-line code (Roe 2002), dividing Titan's atmosphere below $1000 \mathrm{~km}$ into 50 spherical shells evenly spaced in $\log P$ and using spectral bins of $3 \times 10^{-4} \mathrm{~cm}^{-1}$ in order to resolve even the narrowest emission lines. Line parameters for acetylene $\left(\mathrm{C}_{2} \mathrm{H}_{2}\right)$ and $\mathrm{HCN}$ are from the highresolution transmission molecular absorption database (Rothman et al. 1998), with the HCN line positions adjusted to agree with the observations of Duxbury \& Gang (1989). The temperature-pressure profile is the "recommended" profile of Yelle et al. (1997). Scattering effects are ignored, and Titan's haze is modeled as a single layer with a lower cutoff of $50 \mathrm{~km}$ and with haze opacity scale height equal to the gas density scale height. The abundances of $\mathrm{HCN}$ and $\mathrm{C}_{2} \mathrm{H}_{2}$ are each parameterized with their fractional abundances at 1 mbar 

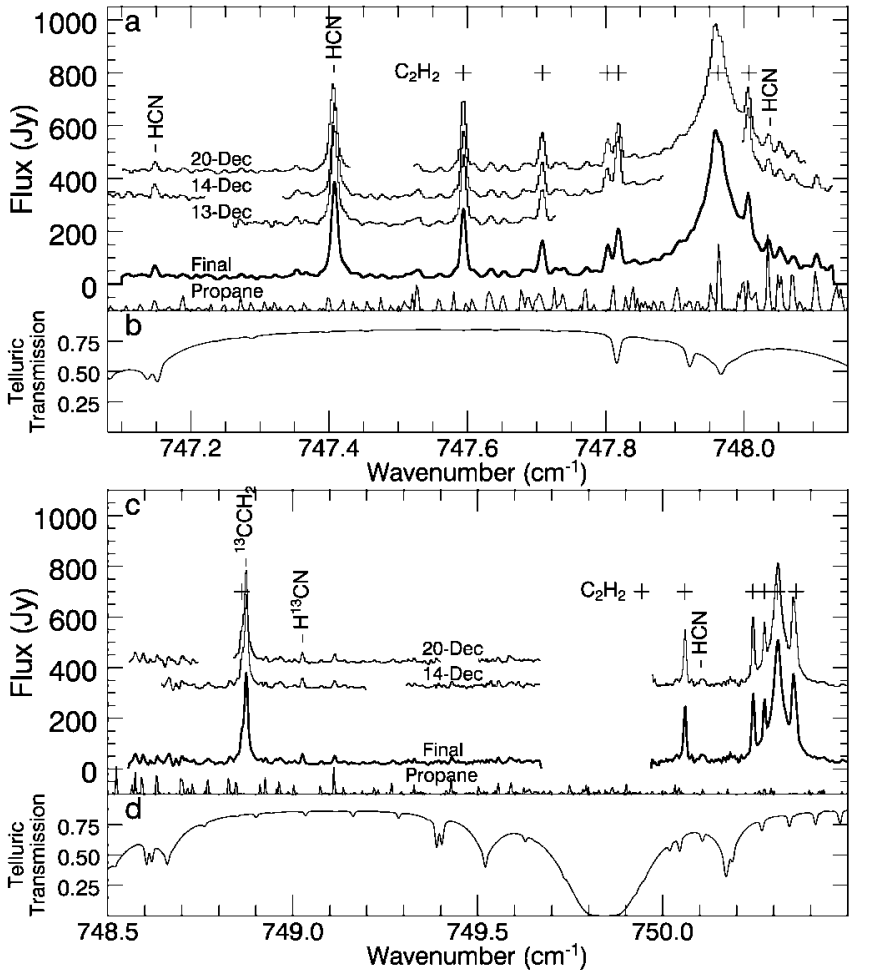

FIG. 1.- $(a, c)$ Reduced spectra from each night (offset for clarity) and a final combined spectrum. $(b, d)$ Calculated telluric transmission (Roe 2002, Appendix A). When compared with an empirical absorption coefficient spectrum of propane (shown at bottom of $[a, c]$ in arbitrary units; see $\S 3$ for more details), nearly all unlabeled features in the observed spectra are seen to be associated with propane.

$\left(F_{\mathrm{HCN}, 1 \mathrm{mbar}}, F_{\mathrm{C}_{2} \mathrm{H}_{2}, 1 \mathrm{mbar}}\right)$ and with the slope of $\log$ (abundance)$\log$ (pressure) $\left(n_{\mathrm{HCN}}, n_{\mathrm{C}_{2} \mathrm{H}_{2}}\right)$, i.e., $F_{\mathrm{HCN}}=F_{\mathrm{HCN}, 1 \mathrm{mbar}}\left(P_{\mathrm{mbar}}\right)^{n \mathrm{HCN}}$.

We investigated both a constant propane vertical profile as well as scaled versions of an abundance profile predicted by photochemical modeling (Lara et al. 1996; Banaszkiewicz et al. 2000). Species are held to saturation vapor pressures below their condensation altitudes. In order to calculate Titan's total flux, the model is run at 32 points from the center of Titan's disk to off the edge of Titan's solid limb, with the calculation points more closely spaced near the limb.

The modeling of propane's emission spectrum requires additional discussion. Several line lists exist for propane's $\nu_{26}$ band at $748 \mathrm{~cm}^{-1}$. These include the GEISA databank (Jacquinet-Husson et al. 1999), an unpublished list based on fits to the laboratory spectra of Nadler \& Jennings (1989) and Hillman et al. (1992; Blass et al. 1988), and a list generated using the code of Typke (1976) and the parameters of Gassler, Reissnauer, \& Huttner (1989). Figure 2 shows calculated spectra using each of these line lists compared to low-resolution laboratory spectra of Giver, Varanasi, \& Valero (1984) and to the high-resolution spectra used in Hillman et al. (1992). Given the poor fit of the calculated spectra to the observed spectra in Figure 2 at the wavenumbers that we observed, we opted to use the low-pressure (1-3 torr) low-temperature $(\sim 175 \mathrm{~K})$ high-resolution $\left(R \approx 3 \times 10^{5}\right)$ transmission spectra of Hillman et al. $(1992)^{5}$ to construct an empirical absorption coefficient $\left(k_{\nu}\right)$ spectrum for propane. This involved fitting for the smoothly

${ }^{5}$ Available at http://diglib.nso.edu/nso_user.html.
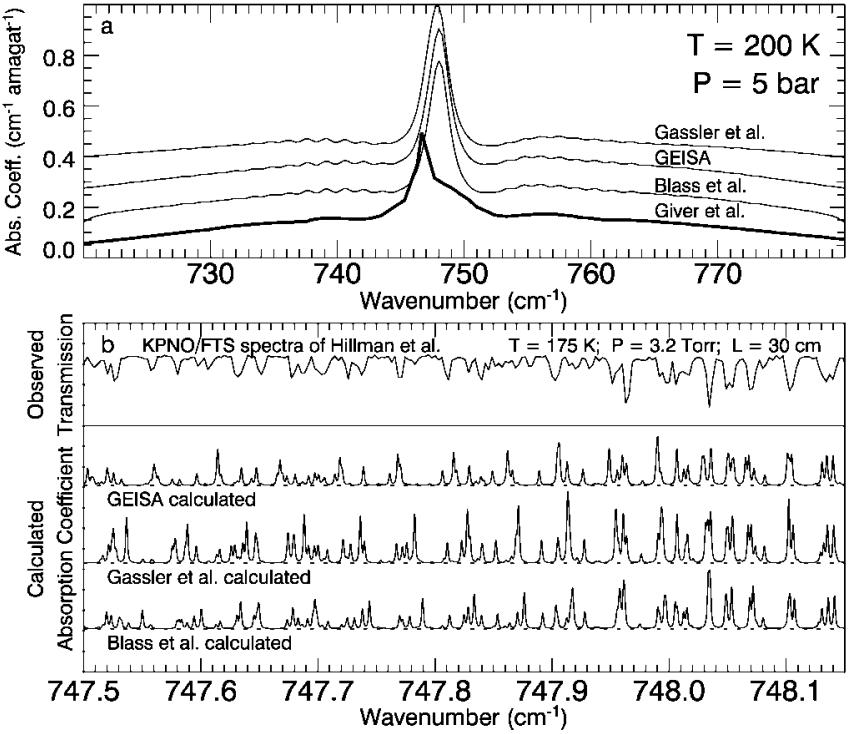

FIG. 2.-(a) Comparison between low-resolution laboratory measurements of Giver et al. (1984) at $200 \mathrm{~K}$ with calculated spectra for the same conditions using the available line lists. Calculated spectra are scaled to match the observed spectrum as closely as possible and offset for clarity. There is good agreement in the wings of the band; however, over $\sim 740-760 \mathrm{~cm}^{-1}$ there is very poor agreement between calculations and observation. (b) Comparison of calculated spectra using the available line lists at $175 \mathrm{~K}$ and 3.2 torr with an observed transmission spectrum of propane at the same conditions from Hillman et al. (1992). There is good agreement at some wavenumbers (e.g., $748.03-748.15 \mathrm{~cm}^{-1}$ ) and poor agreement at others (e.g., $<748.0 \mathrm{~cm}^{-1}$ ).

varying baseline and then converting to $k_{\nu}$ units $\left(\mathrm{cm}^{-1}\right.$ amagat $^{-1}$ ) using the sample's known path length $(30 \mathrm{~cm})$, pressure, and temperature. In using this empirical $k_{v}$ spectrum, we are implicitly assuming that $k_{v}$ is independent of temperature. Our propane observations primarily probe regions of Titan's atmosphere that are at $135-175 \mathrm{~K}$ and are therefore reasonably well matched by the conditions of these laboratory data used to construct $k_{v}$. Most of our spectral fitting was performed using this empirical $k_{v}$ for propane. As a test of the importance of the mismatch between Titan atmospheric and laboratory temperatures, we refitted our data using the Blass et al. line list scaled to fit our empirical $k_{\nu}$ at $175 \mathrm{~K}$, along with the partition sum parameterization of Fischer \& Gamache (2002), to represent propane.

\section{FITTING MODEL TO DATA}

We fitted model to data with an "amoeba"-type minimization while varying six parameters: total haze opacity $\left(\tau_{\text {haze }}\right)$, propane $\left(F_{\mathrm{C}_{3} \mathrm{H}_{8}}\right)$, acetylene $\left(F_{\mathrm{C}_{2} \mathrm{H}_{2}, 1 \text { mbar }}, n_{\mathrm{C}_{2} \mathrm{H}_{2}}\right)$, and $\mathrm{HCN}\left(F_{\mathrm{HCN}, 1 \text { mbar }}, n_{\mathrm{HCN}}\right)$. Figure 3 compares the observed spectrum with the best-fit model with and without propane, in one case using the Blass et al. (1988) line list and in the other using our empirical $k_{\nu}$ to represent propane. The detection of propane is unambiguous, and the bestfit abundance assuming a constant vertical profile and using our empirical $k_{\nu}$ is $6.2 \times 10^{-7}$. For acetylene and HCN, our best fits are characterized by $F_{\mathrm{C}_{2} \mathrm{H}_{2}}=4.4 \times 10^{-6}\left(P_{\text {mbar }}\right)^{-0.45}$ and $F_{\mathrm{HCN}}=$ $3.8 \times 10^{-7}\left(P_{\text {mbar }}\right)^{-0.18}$. The haze is best fitted by $\tau_{\text {haze }}=0.21$, although this value is extremely dependent on the assumed vertical haze profile and should not be overinterpreted.

We found that reasonable fits to the $\mathrm{HCN}$ and $\mathrm{C}_{2} \mathrm{H}_{2}$ features required a nonconstant vertical abundance. Even with our two variable $\log$-log parameterization we see nonzero residuals on 

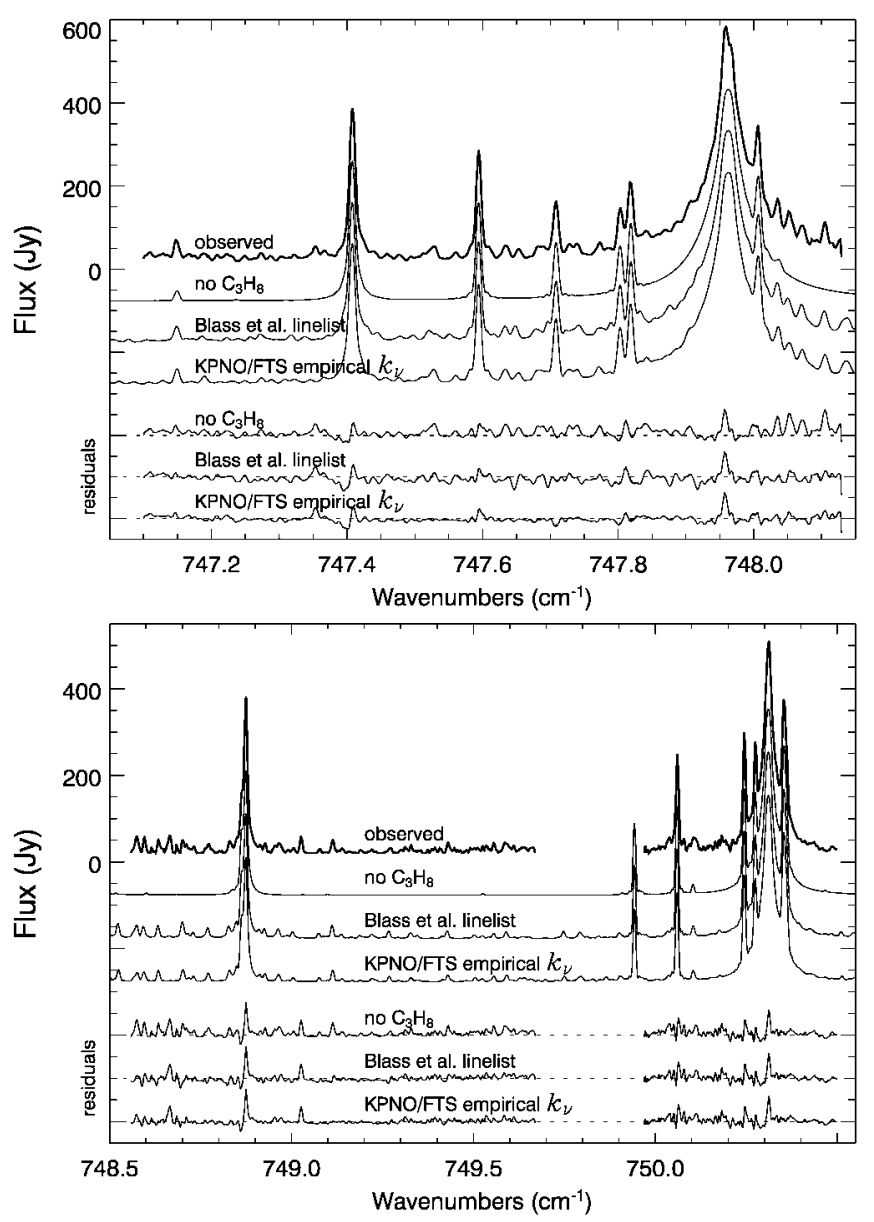

FIG. 3.-Comparison of observed spectrum with model convolved to the observed spectral resolving power. Spectra are offset for clarity, and residuals are shown on the same scale. The best fit is obtained using our empirical $k_{v}$ spectrum for propane.

these features, suggesting a more complicated vertical structure that we will explore further in future work with similar spectra covering many more transitions. Other significant nonzero residuals are explained as an unidentified species at $747.35 \mathrm{~cm}^{-1}$, an imperfectly corrected telluric feature at $748.68 \mathrm{~cm}^{-1}$, not fitting for the ${ }^{13} \mathrm{CCH}_{2} / \mathrm{C}_{2} \mathrm{H}_{2}$ ratio at $748.88 \mathrm{~cm}^{-1}$, and an $\mathrm{H}^{13} \mathrm{CN}$ feature at $749.02 \mathrm{~cm}^{-1}$ that is missing from the model.

Our focus here is propane, and thus we do not present uncertainties on the vertical abundance parameters for $\mathrm{HCN}$ and $\mathrm{C}_{2} \mathrm{H}_{2}$. As is apparent in Figure 1, random errors are negligible in our spectra. The other sources of uncertainty are flux calibration, errors associated with our empirical absorption coefficient method, the extent to which propane abundance is not constant with altitude, the extent to which Titan's temperaturepressure profile deviates from that derived by Yelle et al. (1997), and the extent to which propane's abundance varies with latitude.

The uncertainty in flux calibration is at worst $20 \%$, which maps almost linearly into a $20 \%$ uncertainty in propane abundance. The primary potential error from our empirical absorption coefficient method is the mismatch of laboratory sample temperature $(175 \mathrm{~K})$ to the temperature of the propane in Titan's stratosphere. In our model, spectra greater than $80 \%$ of the propane flux arises from the pressure range 13-0.24 mbar (90$250 \mathrm{~km}$ altitude), where temperatures range over 135-175 K.

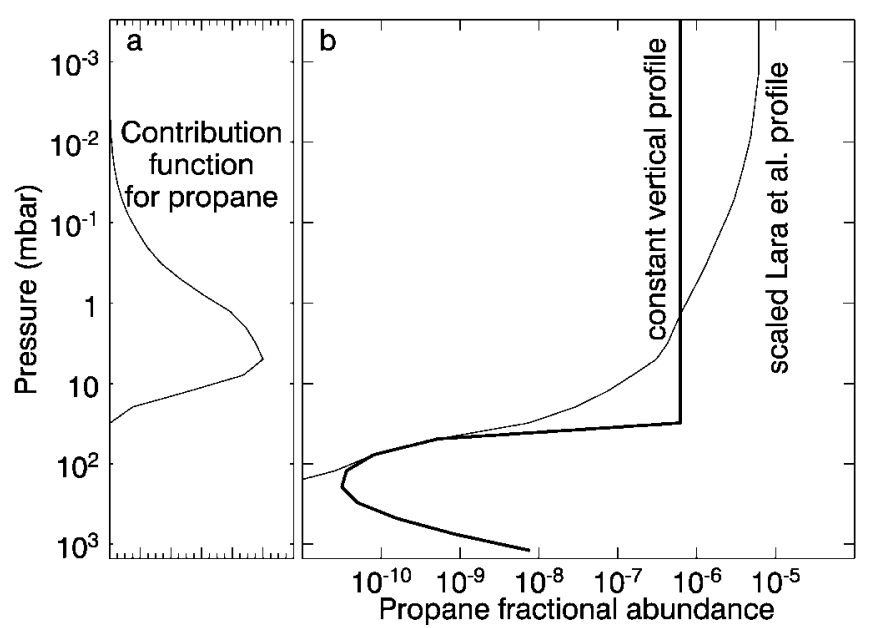

FIG. 4.-(a) Contribution function of propane emission at the middle of the $748.10 \mathrm{~cm}^{-1}$ feature. The contribution function is the partial derivative of propane emission with respect to $\log P$. (b) Comparison of the two best-fit vertical abundance profiles.

To investigate this uncertainty we refitted using the Blass et al. (1988) line list (see Fig. 3). The overall fit is lower quality than with our empirical $k_{v}$, and the best-fit propane abundance is only $1.1 \pm 0.1$ times more.

Recent photochemical models (e.g., Lara et al. 1996; Banaszkiewicz et al. 2000) predict that the propane abundance is relatively constant over $400-850 \mathrm{~km}(11-1.5 \mu \mathrm{bar})$. Above these altitudes propane is depleted by photolysis and reaction with $\mathrm{C}_{2} \mathrm{H}$, while below this range the effect of eddy diffusion and the cold-trap of the tropopause is to reduce the propane abundance. Our observations are primarily sensitive to the pressure range $13-0.24$ mbar (90-250 km altitude), as shown in Figure $4 a$. We refitted our spectra using the model-predicted propane vertical profile and found a best fit when the modelpredicted profile is multiplied by a factor of 2.9. Figure $4 b$ shows this best-fit scaled profile of Lara et al. (1996) along with the best-fit constant vertical abundance profile. The quality of the fits to our data does not distinguish between these two cases.

The uncertainty in the Yelle et al. (1997) temperaturepressure profile is not well quantified, and therefore our reported uncertainties do not account for this source of error. Roughly, a change in stratospheric temperature of $\pm 5 \mathrm{~K}$ requires a $\mp 20 \%$ change in propane abundance.

There is no strong evidence that propane abundance is constant with latitude on Titan. A first attempt at modeling the seasonal variation of photochemistry on Titan (Lebonnois et al. 2001) predicts that propane abundance will vary by a factor of a few from north to south depending on season. With TEXES on an $8-10 \mathrm{~m}$ telescope we would be able to measure the abundance as a function of latitude, which will place new strong constraints on models of seasonal photochemistry.

\section{SUMMARY}

We present the first spectrally resolved detection of propane in Titan's atmosphere. We measure propane's fractional abundance to be $(6.2 \pm 1.2) \times 10^{-7}$, assuming the recommended temperature-pressure profile of Yelle et al. (1997) and a constant abundance with altitude and latitude. Our observations are primarily sensitive to $13-0.24 \mathrm{mbar}$ (90-250 km altitude). 
Alternatively, our data require the predicted propane profile of Lara et al. (1996) to be increased by a factor of 2.9.

The current theoretical understanding of propane's vibrationrotation spectrum is inadequate, and available line lists do not fit our Titan spectra or laboratory propane spectra well. Therefore, we fitted for Titan's propane abundance using an empirical absorption coefficient spectrum derived from low-temperature laboratory spectra of propane.

The most important observational advance that can be made toward detecting new species in planetary atmospheres is to increase the spectral resolving power to $R>$ a few $\times 10^{4}$ such that individual lines are resolved and separated. High spectral resolution allows us to separate the contribution of a minor species (propane) from a strongly emitting species (acetylene).

We thank several people who shared their time and advice, including especially L. Lara, B. Blass, G. Bjoraker, R. Gamache, V. Typke, and the referee. Observations with TEXES are supported by NSF grant AST 02-05518. T. K. G. and M. J. R. were supported by the SOFIA project through USRA grant 8500-98-005.

\section{REFERENCES}

Banaszkiewicz, M., Lara, L. M., Rodrigo, R., López-Moreno, J. J., \& MolinaCuberos, G. J. 2000, Icarus, 147, 386

Becklin, E. E., \& Neugebauer, G. 1967, ApJ, 147, 799

Blass, W. E., Ataken, A., Brault, J. W., Daunt, S. J., Halsey, G. W., Jennings, D. E., \& Reuter, D. C. 1988, The 13.37 Micron Band of Propane, UTMSL Tech. Memo. (Knoxville: Univ. Tennessee)

Cohen, M., Witteborn, F. C., Walker, R. G., Bregman, J. D., \& Wooden, D. H. 1995, AJ, 110, 275

Coustenis, A., Bezard, B., \& Gautier, D. 1989, Icarus, 80, 54

Coustenis, A., Salama, A., Schulz, B., Ott, S., Lellouch, E., Encrenaz, T. H., Gautier, D., \& Feuchtgruber, H. 2003, Icarus, 161, 383

Duxbury, G., \& Gang, Y. 1989, J. Mol. Spectrosc., 138, 541

Fischer, J., \& Gamache, R. 2002, J. Quant. Spectrosc. Radiat. Transfer, 74, 263

Gassler, G., Reissnauer, B., \& Huttner, W. 1989, Z. Naturforsch. A, 44, 316

Giver, L. P., Varanasi, P., \& Valero, F. P. J. 1984, J. Quant. Spectrosc. Radiat. Transfer, 31, 203

Hillman, J. J., Reuter, D. C., Jennings, D. E., Bjoraker, G. L., \& Blass, W. E. 1992, Spectrochim. Acta, 48, 1249

Jacquinet-Husson, N., et al. 1999, J. Quant. Spectrosc. Radiat. Transfer, 62, 205
Lacy, J. H., Richter, M. J., Greathouse, T. K., Jaffe, D. T., \& Zhu, Q. 2002, PASP, 114, 153

Lara, L. M., Lellouch, F., Lopez-Moreno, J. J., \& Rodrigo, R. 1996, J. Geophys. Res., 101, 23,261

Lebonnois, S., Toublanc, D., Hourdin, F., \& Rannou, P. 2001, Icarus, 152, 384

Maguire, W. C., Hanel, R. A., Jennings, D. E., Kunde, V. G., \& Samuelson, R. E. 1981, Nature, 292, 683

Nadler, S., \& Jennings, D. E. 1989, J. Quant. Spectrosc. Radiat. Transfer, 42, 399

Roe, H. G. 2002, Ph.D. thesis, Univ. California, Berkeley

Rothman, L. S., et al. 1998, J. Quant. Spectrosc. Radiat. Transfer, 60, 665

Toublanc, D., Parisot, J. P., Brillet, J., Gautier, D., Raulin, F., \& McKay, C. P. 1995 , Icarus, 113,2

Typke, V. 1976, J. Mol. Spectrosc., 63, 170

Yelle, R. V., Strobell, D. F., Lellouch, E., \& Gautier, D. 1997, in Huygens: Science, Payload and Mission, ed. A. Wilson (ESA SP-1177; Noordwijk: ESA), 243

Yung, Y. L., Allen, M., \& Pinto, J. P. 1984, ApJS, 55, 465 